\title{
Implementation of the Chicago sum frequency laser at Palomar laser guide star test bed
}

Viswa Velur, Edward Joseph Kibblewhite, Richard G. Dekany, Mitchell Troy, Hal L. Petrie, et al.

Viswa Velur, Edward Joseph Kibblewhite, Richard G. Dekany, Mitchell Troy, Hal L. Petrie, Robert P. Thicksten, Gary Brack, Thang Trin, Matthew Cheselka, "Implementation of the Chicago sum frequency laser at Palomar laser guide star test bed," Proc. SPIE 5490, Advancements in Adaptive Optics, (25 October 2004); doi: 10.1117/12.550675

Event: SPIE Astronomical Telescopes + Instrumentation, 2004, Glasgow, United Kingdom 


\title{
Implementation of the Chicago sum frequency laser at Palomar laser guide star test bed
}

\author{
Viswa Velur $^{a, b}$, Edward Kibblewhite ${ }^{b}$, Richard Dekany ${ }^{a}$, Mitchell Troy ${ }^{c}$, Hal Petrie ${ }^{a}$, Robert \\ Thicksten $^{a}$, Gary Brack ${ }^{c}$, Thang Trin ${ }^{c}$, Matthew Cheselka ${ }^{d}$ \\ ${ }^{a}$ Caltech Optical Observatories, Pasadena, CA 91125-24, USA; \\ ${ }^{b}$ Fermi Institute, University of Chicago, Chicago, IL-60637, USA; \\ ${ }^{c}$ Interferometry and Large Optics, Jet Propulsion Lab, 4800 Oak Grove Drive, Pasadena, CA \\ 91109, USA \\ ${ }^{d}$ Custom Scientific Software, Tucson, AZ, USA
}

\begin{abstract}
Work is underway at the University of Chicago and Caltech Optical Observatories to implement a sodium laser guide star adaptive optics system for the 200 inch Hale telescope at Palomar Observatory. The Chicago sum frequency laser (CSFL) consists of two pulsed, diode-pumped, mode-locked Nd:YAG lasers working at 1.064 micron and 1.32 micron wavelengths. Light from the two laser beams is mixed in a non-linear crystal to produce radiation centered at $589 \mathrm{~nm}$ with a spectral width of $1.0 \mathrm{GHz}$ (FWHM) to match that of the Sodium-D2 line. Currently the 1.064 micron and 1.32 micron lasers produce 14 watts and 8 watts of TEM-00 power respectively. The laser runs at $500 \mathrm{~Hz}$ rep. rate with $10 \%$ duty cycle. This pulse format is similar to that of the MIT-Lincoln labs and allows range gating of unwanted Rayleigh scatter down an angle of 60 degrees to zenith angle.

The laser system will be kept in the Coude lab and will be projected up to a laser launch telescope (LLT) bore-sited to the Hale telescope. The beam-transfer optics, which conveys the laser beam from the Coude lab to the LLT, consists of motorized mirrors that are controlled in real time using quad-cell positioning systems. This needs to be done to prevent laser beam wander due to deflections of the telescope while tracking. There is a central computer that monitors the laser beam propagation up to the LLT, the interlocks and safety system status, laser status and actively controls the motorized mirrors.

We plan to install a wide-field visible camera (for high flying aircraft) and a narrow field of view (FoV) IR camera (for low-flying aircraft) as part of our aircraft avoidance system.
\end{abstract}

Keywords: Guide star laser, adaptive optics, large telescope design

\section{INTRODUCTION}

\subsection{The aim of the project}

Laser beacons are the power source that will drive adaptive optics systems of the future, and give ground based telescopes the ability to achieve diffraction limited imaging of faint objects over a wide area of the sky and over fields similar to that of the Hubble Space Telescope. The project aims at building a test facility to mitigate risks in doing laser guide star science for the ELTs, in particular, the Thirty Meter Telescope (TMT) at first light. We are building a test bed to test different sodium laser formats and do head-to-head comparison of their performance in situ. As a first step, we plan to test a laser of unique spectral and temporal format, designed and built at the Fermi Institute at the University of Chicago. This laser is currently being re-assembled in the adaptive optics lab at Palomar Mountain. The authors have designed a Beam Transfer Optics (BTO) system that will facilitate propagation of all laser formats. The Palomar laser guide star test bed will use an all-sky visible camera and a narrow FoV thermal imaging camera for aircraft avoidance.

This paper discusses the status of the project and describes the current state of the AO system, the system architecture of the laser, the beam transfer optics system, and the aircraft avoidance system.

Author to whom correspondence should be sent :

V. V - Robinson 01, Mail Code - 105-24 Caltech, 1200 E California Blvd., Pasadena, CA - $91125 . \quad$ E-mail: vnv@phobos.caltech.edu

Advancements in Adaptive Optics, edited by Domenico Bonaccini Calia, Brent L. Ellerbroek, 


\section{SYSTEM DESIGN AND SUBSYSTEM ARCHITECTURE}

\subsection{Overview of the Palomar adaptive optics system}

The Hale telescope at Palomar has a unique niche to act as a LGS test bed as it has a large Coude lab facility that can be used to test new laser technologies. This will let us decide on optimum characteristics of a sodium guide star laser. The Coude lab facility will be used to align and test new and existing laser concepts with a rugged yet simple BTO system. The Palomar Adaptive Optics (PALAO) system is a high order AO system that gives bright guide star Strehls as high as $80 \%$ at $2.2 \mu \mathrm{m}$. The system can operate at a maximum speed of 2000 $\mathrm{Hz}$ with $7 e^{-} s$ of read noise. The limiting guide star magnitude for the system is $13.5 M_{v}$ with $10-15 \%$ Strehls at $2.2 \mu \mathrm{m}$ at slower frame rates. These are typical measurements are Palomar's median seeing of 1 arc-sec. Figure 1 shows comparison of three images at $K_{s}$ band $(\lambda=2.145 \mu \mathrm{m}$, width=0.3 $\mu \mathrm{m})$. The first is a perfect image as viewed by the AO system with no atomsphere or wave-front distortions, the second one is a image simulated with atomsphere and errors due to finite band-width, finite sampling and photon noise. The third image is one obtained from the PALAO system; this has all the errors accounted for in second image along with errors due to optical alignment and calibration. The images are with a $r_{0}=11 \mathrm{~cm}$ with 5 second exposures. One can see that the Strehl numbers are in excellent agreement to the fact that our calibration error estimates are about $100 \mathrm{~nm}$. The left image in figure 2 shows the comparison of the PSFs obtained from the above mentioned three cases. The image on the right side of figure 2 shows the enclosed energy for the three cases along with that of the seeing limited case. The simulations were done with Caltech's Arroyo AO simulation library ${ }^{1}$

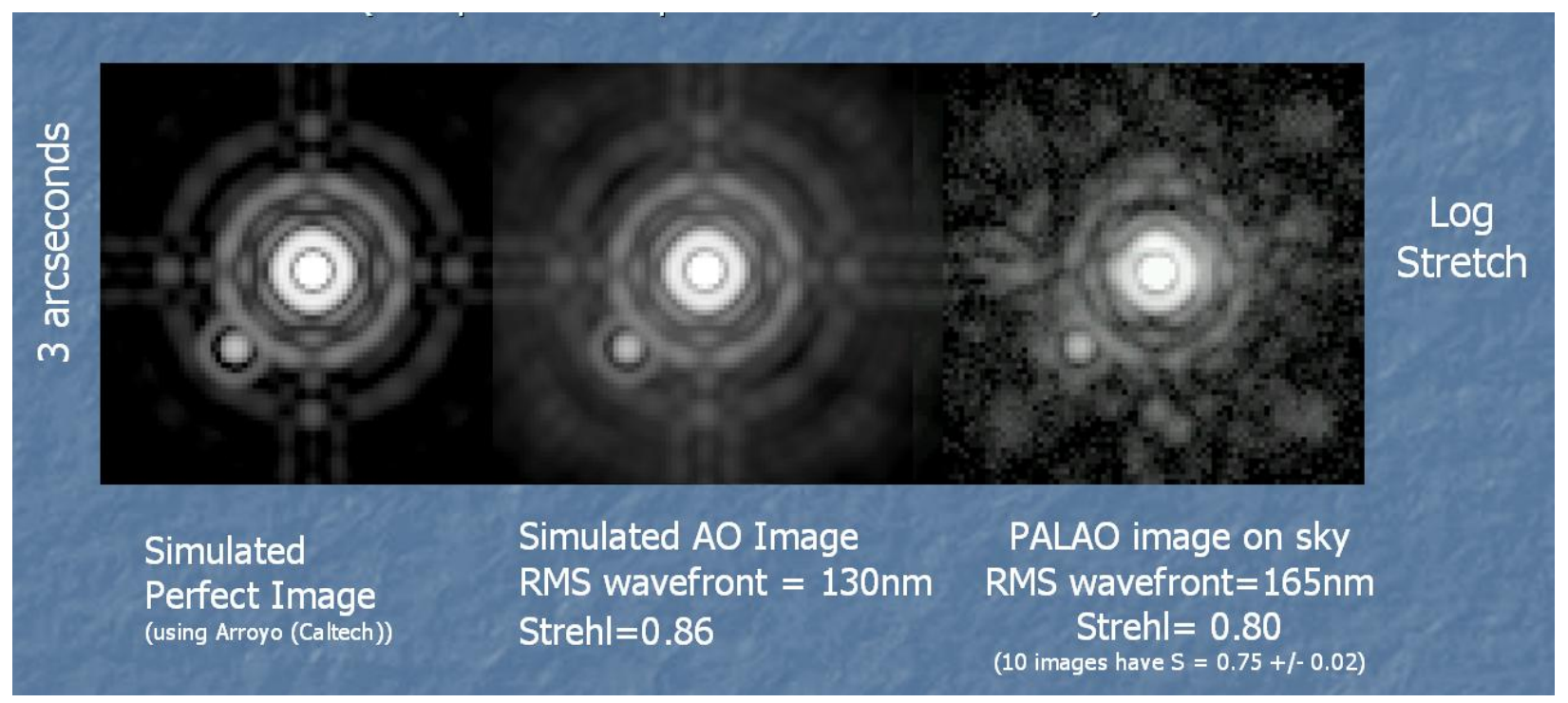

Figure 1. Comparison of simulated image that that obtained on the sky by PALAO

\subsection{Chicago sum frequency laser}

The Chicago Sum Frequency Laser (CSFL) is a diode-pumped, mode-locked, pulsed, all solid-state laser that is based on a prototype built by MIT Lincoln Labs. The CSFL has a micro-macro pulse format based on the article by Milonni et. al. ${ }^{5}$ This sum frequency laser uses two Nd:YAG lasers operating at $1064 \mathrm{~nm}$ and 1319 $\mathrm{nm}$ to produce $589 \mathrm{~nm}$ radiation by mixing them in a non-linear crystal external to the laser cavities. The source lasers at $1064 \mathrm{~nm}$ and $1319 \mathrm{~nm}$ are built to have a line-width of about $1.5 \mathrm{MHz}$ and $1.15 \mathrm{MHz}$ to produce $589 \mathrm{~nm}$ light with a line-width of $0.9 \mathrm{MHz}$. Figure 3 shows the schematic of the laser. The basic laser cavity design of either laser is based on classic paper by Magni. ${ }^{4}$ Both the source laser cavities have flat high reflector (HR) and output coupler (OC) with a lens in between. The gain medium, Nd:YAG material, is placed such 

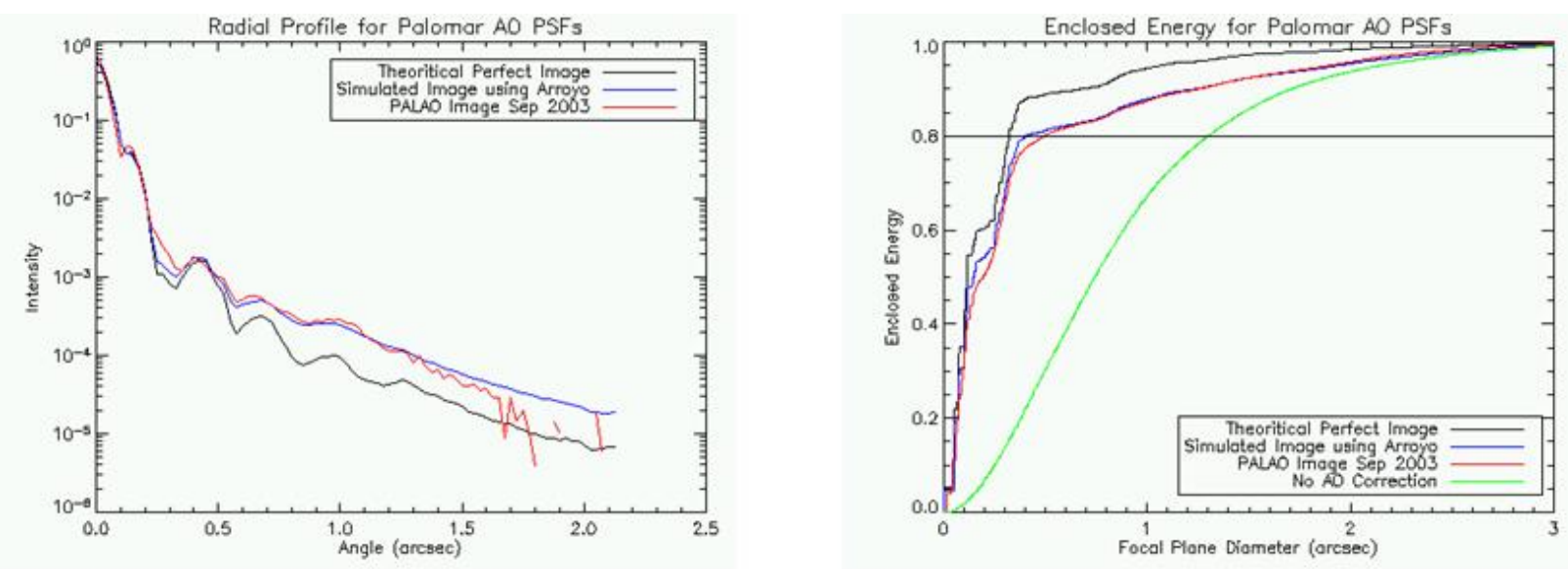

Figure 2. Comparison of theoritical AO PSFs and enclosed energy between simulation and PALAO

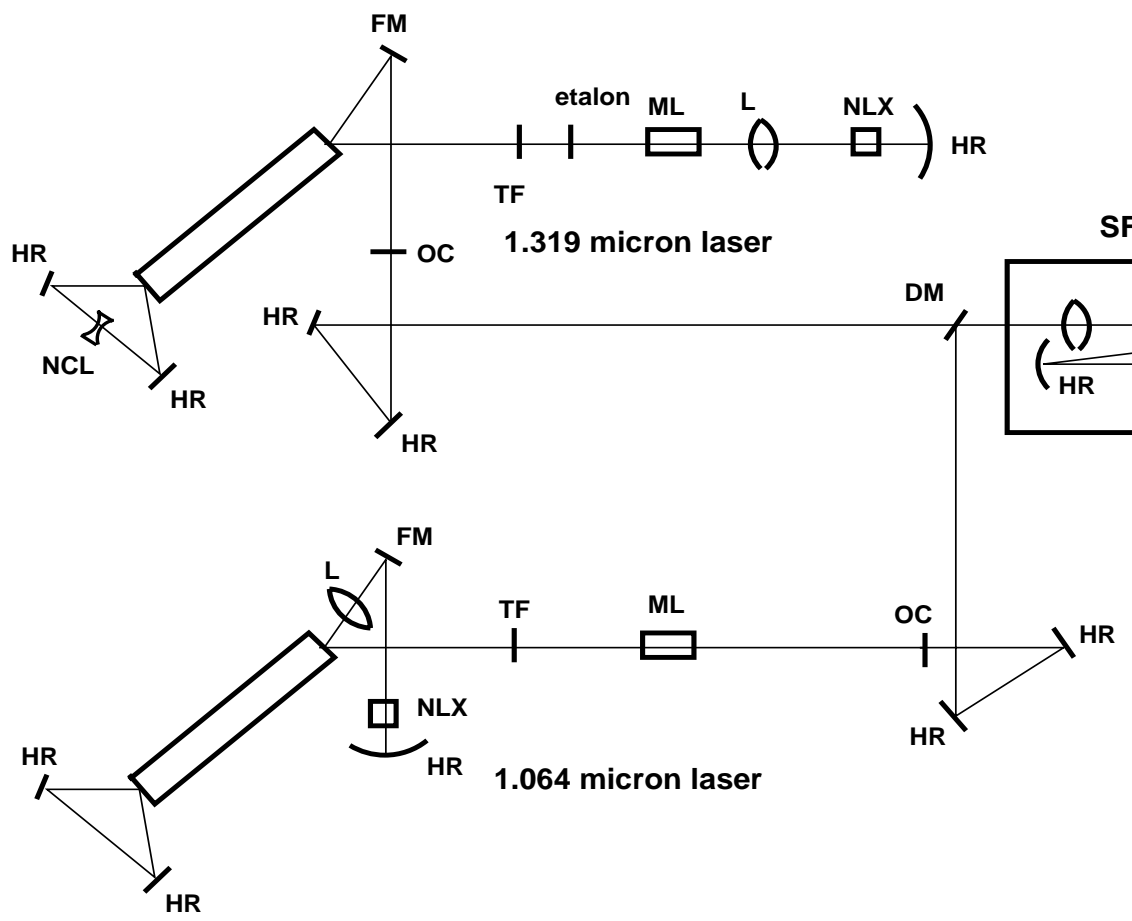

DM - dichroic mirror

FM - Fold mirror

HR - high reflectance mirror

$\mathrm{L}$ - lens

ML - modelocker

NLX - non-linear crystal

OC - output coupler

SFG - sum freq. generation

TEC - thermo-electric cooler

TF - tuning filter

NCL - negative cylindrical lens

Figure 3. The Chicago sum frequency laser 
as to match the TEM-00 mode based on Magni's theory to the fluorescence obtained from the $808 \mathrm{~nm}$ pump diodes. The authors have engineered a new gain module with superior heat transfer characteristics and small area of $808 \mathrm{~nm}$ pump light concentration for the $1319 \mathrm{~nm}$ laser. This new gain module is optimally located where the theoritical beam ${ }^{4}$ matches the diode pump cross-section with appropriate thermal lens compensation provided by a negative cylindrical lens in the cavity. There is a non-linear crystal in both source laser cavities to suppress relaxation oscillations, thus generating almost a rectangular laser pulse. The tuning filters help set the line-width of the two lasers. There is extra Fabry-Perot etalon in the $1319 \mathrm{~nm}$ laser to prevent any 1064 $\mathrm{nm}$ laser action in the cavity. The cavity length of both lasers is $1.5 \mathrm{~m}$, hence setting cavity mode spacing to $100 \mathrm{MHz}$. The mode-lockers have Brewster angled facets so as to minimize reflection losses in the desired polarization direction. The modelocker electronics consist of a signal generator, a pre-amp., two amplifiers and a phase-shifter. The acousto-optic modulator tuned to half the cavity mode spacing. This modulator has the effect of periodically resetting the phase of all the modes to zero every transit time of the cavity. All the modes then add together coherently and the output power is initially increased by a factor of $\mathrm{N}$, where $\mathrm{N}$ is the number modes the cavity supports. $\mathrm{N}$ can be determined by $N=\frac{\nu_{Y A G}}{(c /(2 L))}$. But, after a time of order $\frac{1}{N \nu_{\text {cavity }}}$, the different modes destructively interfere. The result is that the laser generates a pulse train of frequency $\nu_{\text {cavity }}$ and pulse duration $\frac{1}{N \nu_{\text {cavity }}}$. The peak power of the pulse is $\mathrm{N}$ times the power of the non-mode-locked pulse. This is done to increase the sum frequency conversion efficiency by the same amount. The temporal profile of the micro-pulses is transform limited. In December of 2003, we had a single LBO crystal through which we passed the source laser beam twice to get $3.5 \mathrm{~W}$ of TEM-00 light centered at $589 \mathrm{~nm}$ with $1 \mathrm{GHz}$ line-width. We now have new LBO crystals with which we can increase the $589 \mathrm{~nm}$ light output by measuring the beam source laser beam diameters and matching them inside the Sum Frequency Generation (SFG) module using appropriate telescopic lens combination. The new SFG module consists of three LBO crystals on a copper substrate with one lens and two spherical mirrors to focus the beam inside the 3 crystals as shown in figure 3 . The temperature of the substrate is controlled by a thermo-electric cooler with a PID controller to regulate the temperature. The whole SFG module will be enclosed in an air-tight chamber and the pressure inside will be regulated to eliminate any phase mis-match between the beams due to dispersion. Currently our $1064 \mathrm{~nm}$ and $1319 \mathrm{~nm}$ put out 14 $\mathrm{W}$ and $8 \mathrm{~W}$ of avg. power. We expect this new SFG module to generate $8 \mathrm{~W}$ of radiation centered at 589 $\mathrm{nm}$. After transmission losses at BTO and atmosphere, we expect to shine greater than $5 \mathrm{~W}$ of light tuned to sodium D2 light onto the mesosphere. Our $1064 \mathrm{~nm}$ and $1319 \mathrm{~nm}$ beams have excellent beam quality with $M^{2}$ of 1.1 as shown in figure 4. The figure also shows rectangular laser pulse with relaxation oscillations suppressed, modelocked pulses and $589 \mathrm{~nm}$ light generated with a single LBO crystal after a single pass through it.

In past experiences at the Dunn Solar Telescope, $1.1 \mathrm{~W}$ of this laser format projected on the sky as been shown to generate a $9.5 M_{v}$ star. $^{3}$ Spot size of 0.9 arc-sec. in 1 arc-sec. seeing was obtained during these tests. We expect the PALAO-LGS image quality to be similar.

\subsection{Beam Transfer Optics}

We plan to propagate the laser along the Coude axis of the telescope. Figure 5 shows the laser beam path from the Coude lab to the laser launch telescope. The Hale telescope is an equatorially mounted telescope; hence if the laser beam is propagated along the polar axes, we won't need to walk the beam as the telescope tracks. But the 200 inch telescope has a high slenderness ratio hence it deflects while tracking. To take out this effect and keep the laser beam bore-sited to its intended path we have a low band-width (about $1 \mathrm{~Hz}$ speed) control using motorized mirrors and quad-cells. One can see a $10 \mathrm{~m}$ long trolley with a moving mirror in figure 5 . This mirror moves along the length of the trolley and rotates to pick of the laser and propagate it through the rest of the beam transfer optics train. The position of this mirror along the length of the trolley and its angular orientation is a function of the telescope DEC angle. Mirrors M1, M2, M3 are motorized and are controlled by a PC based on quad-cell signals from QC2, QC3, and QC4. The DEC angle information comes to the control computer from the telescope control computer (TCS) through a socket connection. The laser bore-siting is done using A/D input channels that read the beam position off the quad-cells and move the mirror in front of the quad-cell in the optics train to account for any deflection in the main telescope structure. The laser is finally fed to a laser launch telescope (LLT) by a fast tip-tilt mirror (FTT). The LLT is mounted on the prime focus cage behind the secondary mirror of the telescope with its axis is parllel with that of the telescope. The optical design of the LLT and mechanical throw of the FSM facilitate pointing the laser beam anywhere inside a field of 

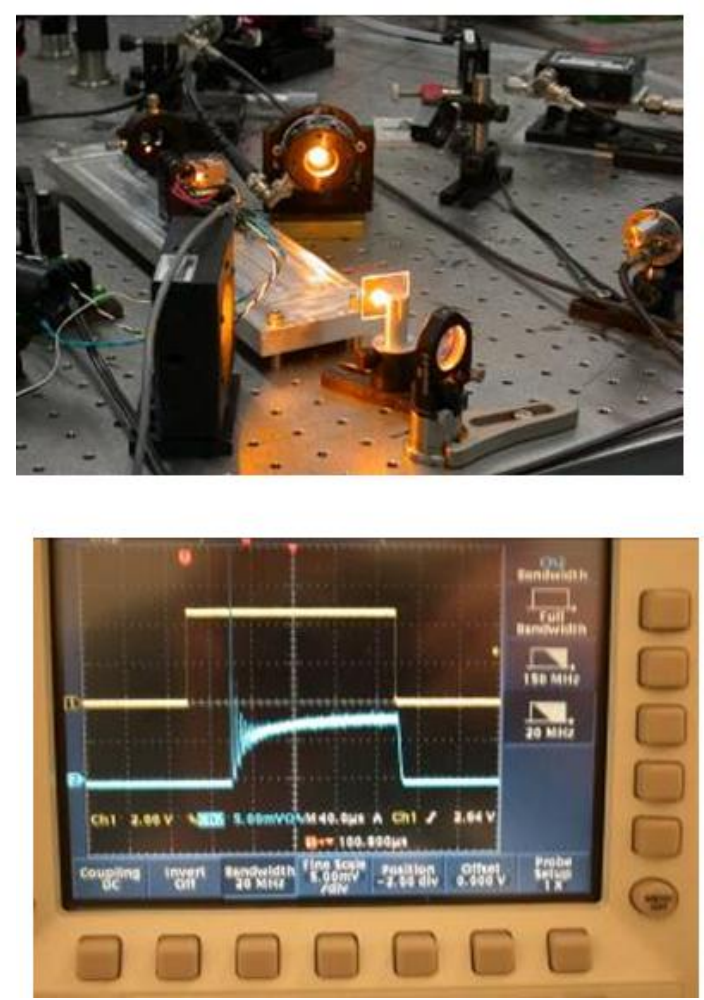
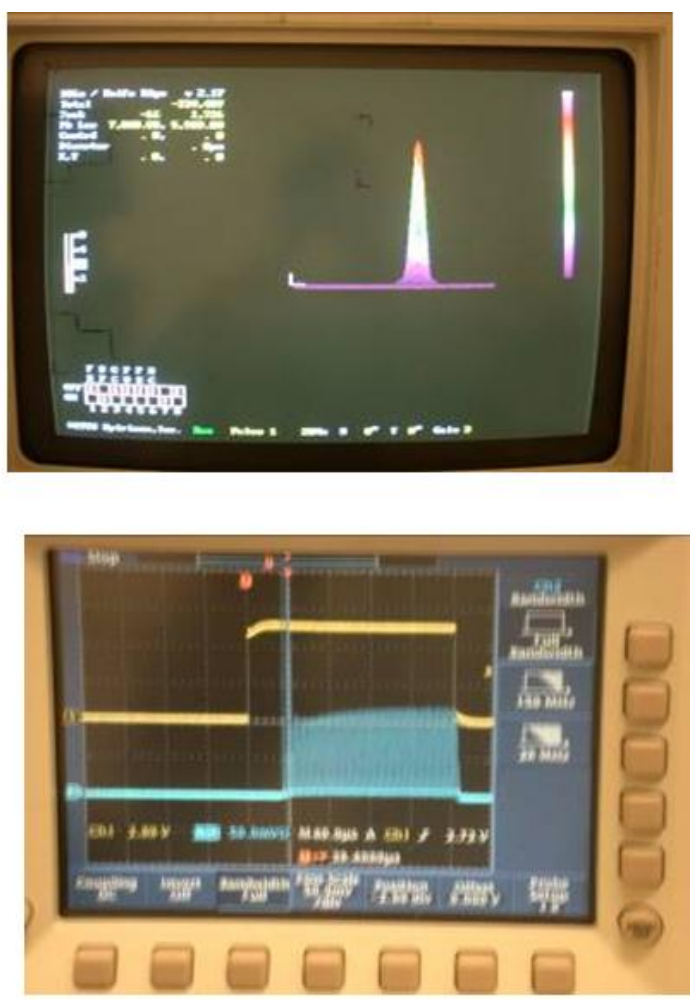

Figure 4. The four figures, in clockwise order from top-left, show the generation of $589 \mathrm{~nm}$ light with one crystal with a single pass, typical beam profile of our source lasers with $M^{2}=1.1$, generation of a rectangular laser pulse with relaxation oscillations suppressed, modelocked laser pulse showing transform limited micro-pulses

3 min diameter. The LLT itself is an $\mathrm{f \# 2}$ system with a parabolic lightweight Hextek primary of $0.5 \mathrm{~m}$ dia. and a Mangin secondary of $38.1 \mathrm{~mm}$ dia. The primary sits on a 6 point Whiffletree mounted on flex-pivots. There are counterweights as shown in figure 6 as seen in other variable orientation mirror mounts for large optics. ${ }^{6}$ Both the primary and the secondary mirrors have motorized control to tip, tilt and piston for easy alignment. The actuators on the secondary mirror are piezo-electric so as to allow precise focusing and aligning of the LLT.

\subsection{Aircraft Avoidance System and other safety systems}

Our aircraft avoidance system uses a narrow field of view thermal imaging camera and a large field of view visible camera as shown in figure 7 . The thermal imaging camera is a LWIR (10 micron range) camera with an $11 \times 8$ degrees FoV with an un-cooled micro bolometric sensor array of $320 \times 240$ pixels. Our visible camera is a 1024 $\times 1024$ pixel CCD array with 2 stage thermo-electric cooling with extra water-cool option. This camera is fitted with a Nikon fish-eye lens to give it a FoV of 180 degrees. The all-sky visible camera will be mounted on top of the telescope dome, so it can view the entire sky. The thermal imaging camera will be placed in the prime focus cage close to the laser beam path. The aircraft detection algorithm ${ }^{2}$ maps vectorially the bright spots seen by the all-sky visible camera and with 3 -sec. exposures and comparing successive frames determines if an aircraft is bound to come into a pre-defined region of risk. The bolometric array is read out at a much faster rate ensuring detection of low-flying aircraft. The flux between successive images is used to detect an aircraft in its field. When the thermal imaging camera senses an aircraft in the region it sends out a TTL signal to shutter the laser. The all-sky camera shutter signal is sent over Ethernet to a computer which in turn shutters the laser.

The observatory peronnel have identified a number of safety issues that include failsafe laser shutter, deadman switches, door interlocks and laser curtains. These have been installed and connected in a Daisy-chain to 


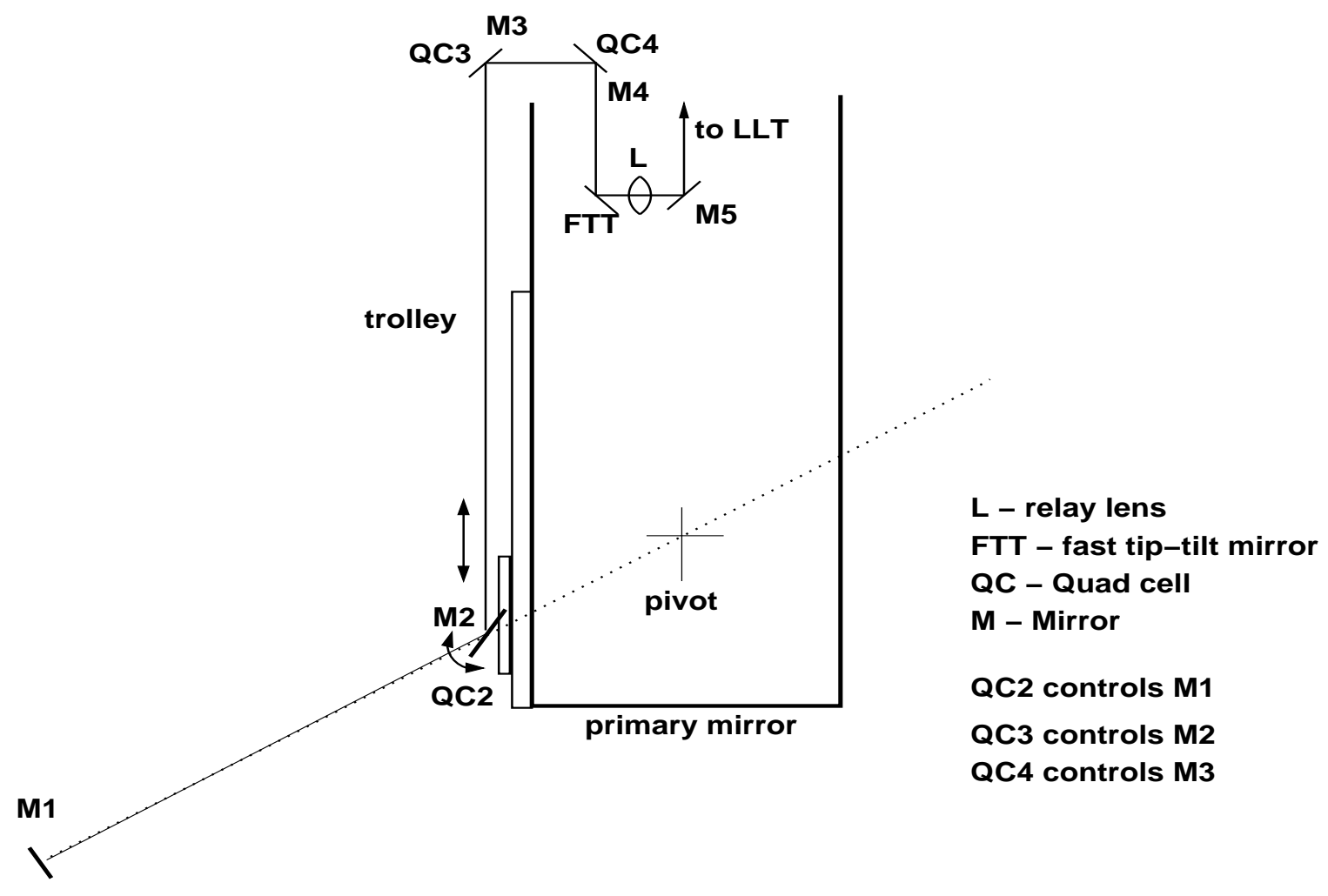

Figure 5. Schematic of the beam transfer optics system

shutter the laser through a hard-wire connection. Sufficient training is being provided to the observatory staff to work with the laser and maintain standards conforming to OSHA laser safety.

\section{SUMMARY AND FUTURE WORK}

Following tests in Chicago in Dec. 2003, where $3.5 \mathrm{~W}$ of $1 \mathrm{GHz}$ light centered at $589 \mathrm{~nm}$ was obtained with stable output of the same over $50 \mathrm{hrs}$ with little operator intervention; we have upgraded the laser, designed and built an aircraft avoidance system, and a BTO system. We will test the BTO components in Palomar in July 2004. A laser launch telescope has been designed and built, that will soon undergo alignment and testing in Palomar. In Oct. 2004, we will have a fully developed test bed to project laser beams up to generate tight laser beacons up at the mesosphere. The Coude infrastructure at Palomar has been improved to house multiple lasers and do head-to-head comparison of return from different lasers.

\section{ACKNOWLEDGMENTS}

This work was supported by the Thirty Meter Telescope (TMT) project and the Center for Adaptive Optics (CfAO). We express our thanks to the engineers and technicians at Palomar, Caltech and Fermi Institute for helping out at various stages of the project and providing useful insights. The authors also thank Corrine Boyer at Gemini Observatory for useful inputs on AAS system design.

The Thirty Meter Telescope (TMT) Project is a partnership of the Association of Universities for Research in Astronomy (AURA), the Association of Canadian Universities for Research in Astronomy (ACURA), the California Institute of Technology and the University of California. The partners gratefully acknowledge the support of the Gordon and Betty Moore Foundation, the US National Science Foundation, the National Research Council of Canada, the Natural Sciences and Engineering Research Council of Canada, and the Gemini Partnership. The 


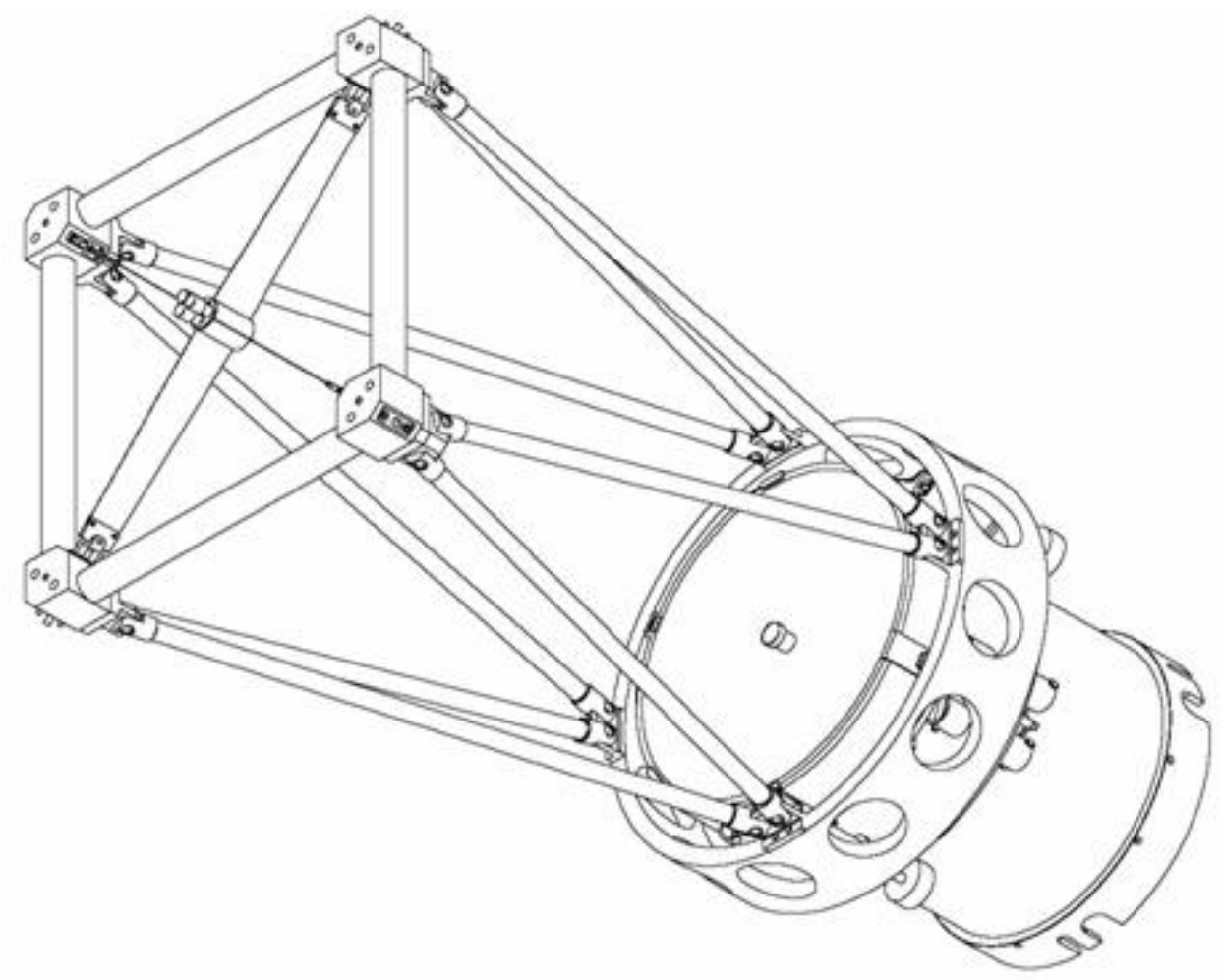

Figure 6. The laser launch telescope

AAS architecture:

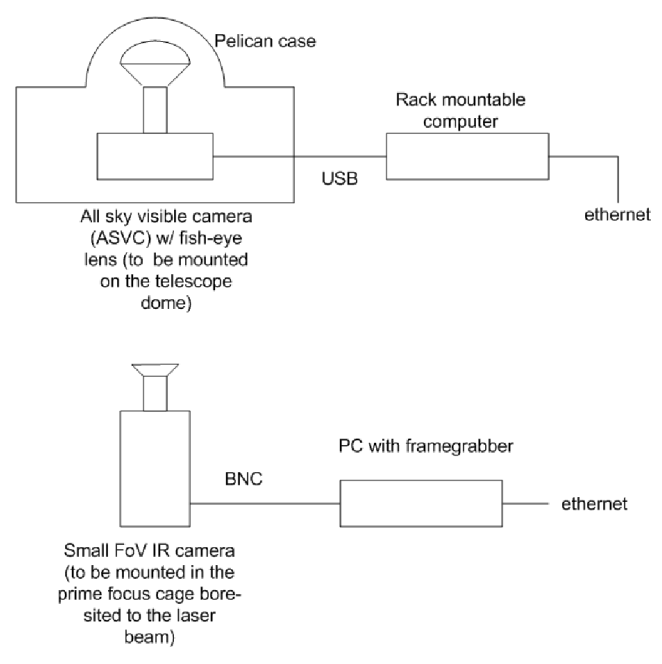

Figure 7. Aircraft avoidance system architecture 
Center for Adaptive Optics (CfAO) is a Science and Technology Center (STC) funded by the National Science Foundation.

\section{REFERENCES}

1. M. Britton, Arroyo AO simulation library, http://eraserhead.caltech.edu/arroyo/arroyo.html.

2. M. Lloyd-Hart, M. Cheselka, R. Hanson, "Verification of a System to Prevent Aircraft Illumination by Adaptive Optics Laser Beacons", 1999 PASP, 111, Pg. 1577.

3. E.J. Kibblewhite, Private communication.

4. V. Magni, "Multielement stable resonators containing a variable lens", JOSA A, Vol. 4, No. 10, Pg. 1962.

5. P.W. Milonni \& L.E. Thode, "Theory of mesospheric sodium fluorescence excited by pulse trains", Applied optics, Vol. 31, No. 6, Pg. 785.

6. P.R. Yoder, "Opto-Mechanical system design", Second Edition, Marcel Dekker Inc., NY. Pg. 407. 